EPJ Web of Conferences 79, 03013 (2014)

DOI: $10.1051 /$ epjconf/20147903013

(C) Owned by the authors, published by EDP Sciences, 2014

\title{
HelioTrope: An innovative and efficient prototype for solar power production
}

\author{
George Papageorgiou ${ }^{1}$, Athanasios Maimaris ${ }^{2}$, Savvas Hadjixenophontos ${ }^{3}$ and Petros Ioannou ${ }^{4}$ \\ ${ }^{1}$ European University Cyprus, 6 Diogenes St, Engomi, CY-2404 Nicosia, Cyprus \\ ${ }^{2}$ Cyprus College, 6 Diogenes St, Engomi, CY-2404 Nicosia, Cyprus \\ ${ }^{3}$ High Efficiency Renewable Energy Ltd, Nicosia, Cyprus \\ ${ }^{4}$ University of Southern California, Los Angeles, California, USA
}

\begin{abstract}
The solar energy alternative could provide us with all the energy we need as it exist in vast quantities all around us. We only should be innovative enough in order to improve the efficiency of our systems in capturing and converting solar energy in usable forms of power. By making a case for the solar energy alternative, we identify areas where efficiency can be improved and thereby Solar Energy can become a competitive energy source. This paper suggests an innovative approach to solar energy power production, which is manifested in a prototype given the name HelioTrope. The Heliotrope Solar Energy Production prototype is tested on its' capabilities to efficiently covert solar energy to generation of electricity and other forms of energy for storage or direct use. HelioTrope involves an innovative Stirling engine design and a parabolic concentrating dish with a sun tracking system implementing a control algorithm to maximize the capturing of solar energy. Further, it utilizes a patent developed by the authors where a mechanism is designed for the transmission of reciprocating motion of variable amplitude into unidirectional circular motion. This is employed in our prototype for converting linear reciprocating motion into circular for electricity production, which gives a significant increase in efficiency and reduces maintenance costs. Preliminary calculations indicate that the Heliotrope approach constitutes a competitive solution to solar power production.
\end{abstract}

\section{Introduction}

In the face of limited fossil fuel energy reserves, rising population trends, unprecedented economic, social, demographic and cultural changes, as well as, disastrous climate changes and losses in the biodiversity, the importance of renewable sources of energy becomes a matter of life or death for humanity. The need for sustainable development becomes obvious if we look at the latest measurements of $\mathrm{CO} 2$ concentration in the atmosphere. According to NOAA (U.S. National Oceanic and Atmospheric Administration) [1] the daily mean of $\mathrm{CO} 2$ concentration exceeded $400 \mathrm{ppm}$ at Mauna Loa, Hawaii on the 9th May, 2013 for the first time ever since the first readings in 1958. This is also backed by recent increase in frequency of environmental disasters worldwide. It is encouraging that leaders around the world began to realise the seriousness of earth's environmental situation. For example it is quite plausible that the mayor of New York Michael Bloomberg attributed the cause of the recent hurricane

This is an Open Access article distributed under the terms of the Creative Commons Attribution License 4.0, which permits unrestricted use, distribution, and reproduction in any medium, provided the original work is properly cited. 
Sandy to human caused destruction of the environment [2]. In unveiling his plans to protect New York from climate change he declared: "In fact, because of rising temperatures and sea levels, even a storm that's not as large as Sandy could, down the road, be even more destructive." The challenge to be tackled is how do we produce and use energy related to consequences to the environment.

On the other hand we have a long way to go until we reach the desired levels of sustainable development. The solar energy alternative could provide us with all the energy we need. As revealed by Crabtree and Lewis [3] the sun gives the earth $10^{17}$ Jules of energy per second. In fact the sun supplies in $11 / 2$ days energy that is equivalent to all the earths' ultimate recoverable resource of oil energy. The challenge is how to capture this vast amount of energy and utilize for our purposes.

Solar energy is a virtually unlimited clean source of energy that remains underutilized even though it has the potential to cover a significant portion of the power needs of the whole planet. The basic requirement for such an ambitious goal is the development of innovative and integrative solutions for solar energy production and storage.

We therefore propose a holistic approach named "Heliotrope" to solar energy production by which we can achieve: Technological development and innovation of know-how in generating electricity from solar energy; Optimization of processes in the production of electricity from solar energy; Improvement of the innovativeness and competitiveness of solar energy systems in relation to other methods of energy production; Increase of the efficiency of capturing solar energy and converting it to other forms of usable energy.

The proposed approach is demonstrated with the design and implementation of a prototype solar energy system (named Heliotrope) for the generation of electricity and other forms of energy for storage or direct use. Forms of clean energy storage include methods and technologies such as: hydrogen (stored as compressed gas or metal hydrides), hot water generation and molten salt bath. The envisioned prototype involves an innovative Stirling engine design and a parabolic concentrating dish with a sun tracking system to maximize efficiency. Further, it utilizes a patent developed by the authors [4] where a mechanism is designed for the transmission of reciprocating motion of variable amplitude into unidirectional circular motion. This is employed in our prototype for converting linear reciprocating motion into circular for electricity production, which gives a significant increase in efficiency and reduces maintenance costs. Preliminary calculations indicate that the proposed Heliotrope approach constitutes a competitive solution to energy production.

\section{The HelioTrope approach to solar power production}

The undeniable fact though is that energy is ample around us and is unlimited and indestructible. Energy simply changes forms and is just a matter of good management practice as how to use it efficiently and effectively.

Especially Solar Energy as noted earlier represents a viable alternative as it is the cleanest and safest form of Energy and exists in vast quantities everywhere around us. The problem of utilizing the opportunity that solar energy provides is that we tend to stick to the old ways of the past, which unfortunately lead to an unsustainable growth with disastrous consequences. The call here is to find innovative ways of transforming and using solar energy so it can directly compete with fossil fuels.

This paper suggests an innovative prototype for efficient and competitive solar energy utilization, which is a major step towards clean energy and the ultimate goal for sustainable development. The prototype is developed based on a holistic approach to solar power production [5] and is depicted below in Fig. 1.

After realizing the need for clean energy production, the first step is to evaluate the current methods of solar energy capture and conversion. This is followed by identifying efficient processes to be included in an integrated model for energy management. The suggestion here is to evaluate the model via simulation experiments before proceeding to the development of a real prototype. Then, the prototype 


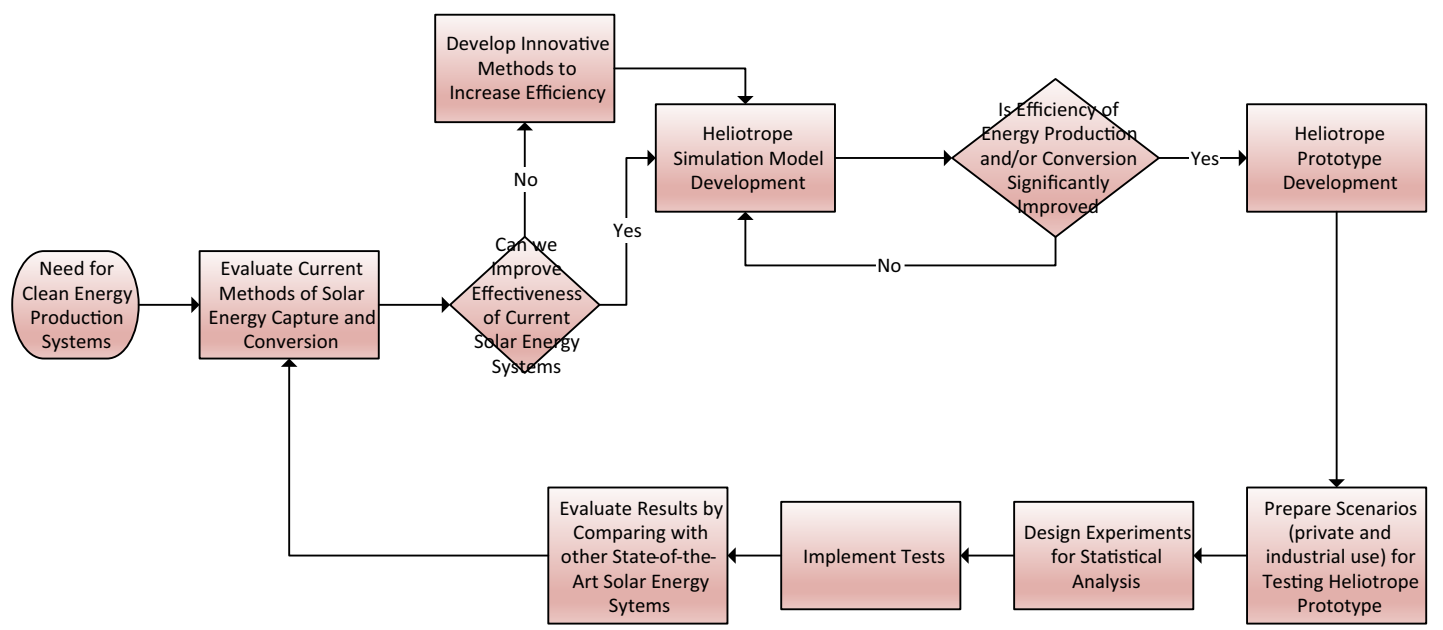

Figure 1. The HelioTrope Holistic Approach to Solar Energy Production.

should be tested in the field via real experiments with statistical analysis so that it proves its capability to be an efficient solar energy system. We are now at the development stage of the HelioTrope prototype which we describe in the next section.

\section{The HelioTrope prototype development with preliminary results}

The HelioTrope prototype, which is shown in Fig. 2 incorporates all the previously analysed solar energy production and conversion parts and technologies into an integrated system. The prototype includes the innovations or improvements that have been identified, developed and tested during the simulation model development as well as synergies associated with the integration of the various parts such as the DC generator, batteries, inverter and electricity management. Further the whole system is to be controlled via the internet where information about its performance will be delivered on a web site or on a mobile phone.

As shown in Fig. 2, the Heliotrope prototype includes a $60 \mathrm{~cm}$ aluminium parabolic dish with a small Stirling engine in the centre. The dish is motorized in two axes in order to increase agility and maximise the incident radiation. In tests carried out by the authors, the hot end temperature of the Stirling engine reached 315 degrees Celsius and the cold end 45 degrees, giving a maximum theoretical efficiency of $46 \%$ as shown in Eq. (1).

$$
\eta=1-\frac{T_{c}}{T_{h}}=1-\frac{273+45}{273+315}=0.46 .
$$

We are now in the process of developing a new HelioTrope prototype which will be able to reach higher working temperatures with the use of new ceric materials.

The first main component of the HelioTrope prototype is the concentrator dish which is shown in design form with its mirrors in Fig. 3. Important to note here is the manufacturing method for producing mirrors for the concentrator dish. Taking into consideration the constraints in the production of mirrors an objective function is developed that minimizes the cost and maximize the performance of the concentrator dish. Constraints such as materials, machines, labour, shape of mirrors, and coating for dust avoidance are taken into account. 


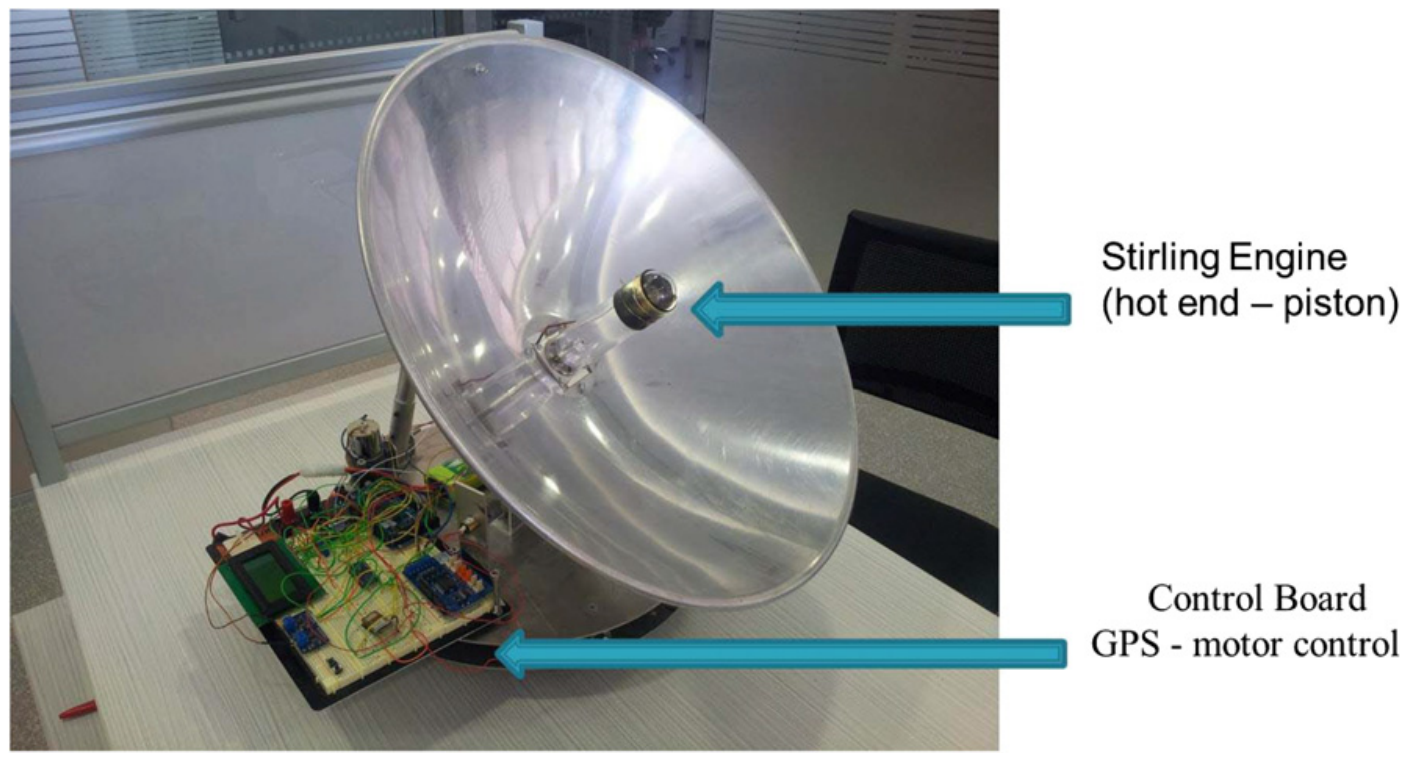

Figure 2. The HelioTrope Prototype.

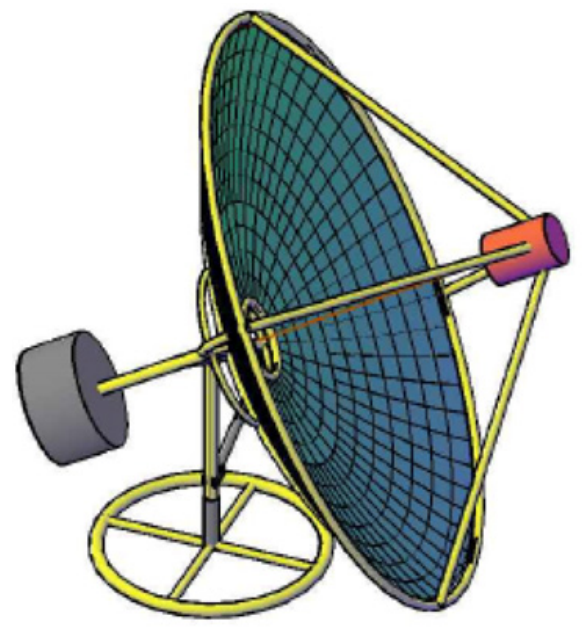

Figure 3. Design of Parabolic Concentrator Dish.

The second main component of the HelioTrope prototype is the Sun Tracker system, which is shown in Fig. 4. Here an implementation of an efficient algorithm [6] was carried out where the concentrator dish tracks the sun movement in order to receive the highest possible energy at the time of day and time of the year. Parameters such as elevation from sea level, location on the earth are taken into account in order to calculate the optimum position of the solar dish in terms of azimuth and elevation angle

Specifically, the controller is tracking the sun incorporates the SPA algorithm [7] using data (location, altitude and time) from a GPS receiver and thus is unaffected by passing clouds. It is based on a closed control loop feedback control process with two optical encoders. The calibration is automated by cantering on the sun and reading the encoders at that instant. There are also a number of failsafe 
$3^{\text {rd }}$ European Energy Conference

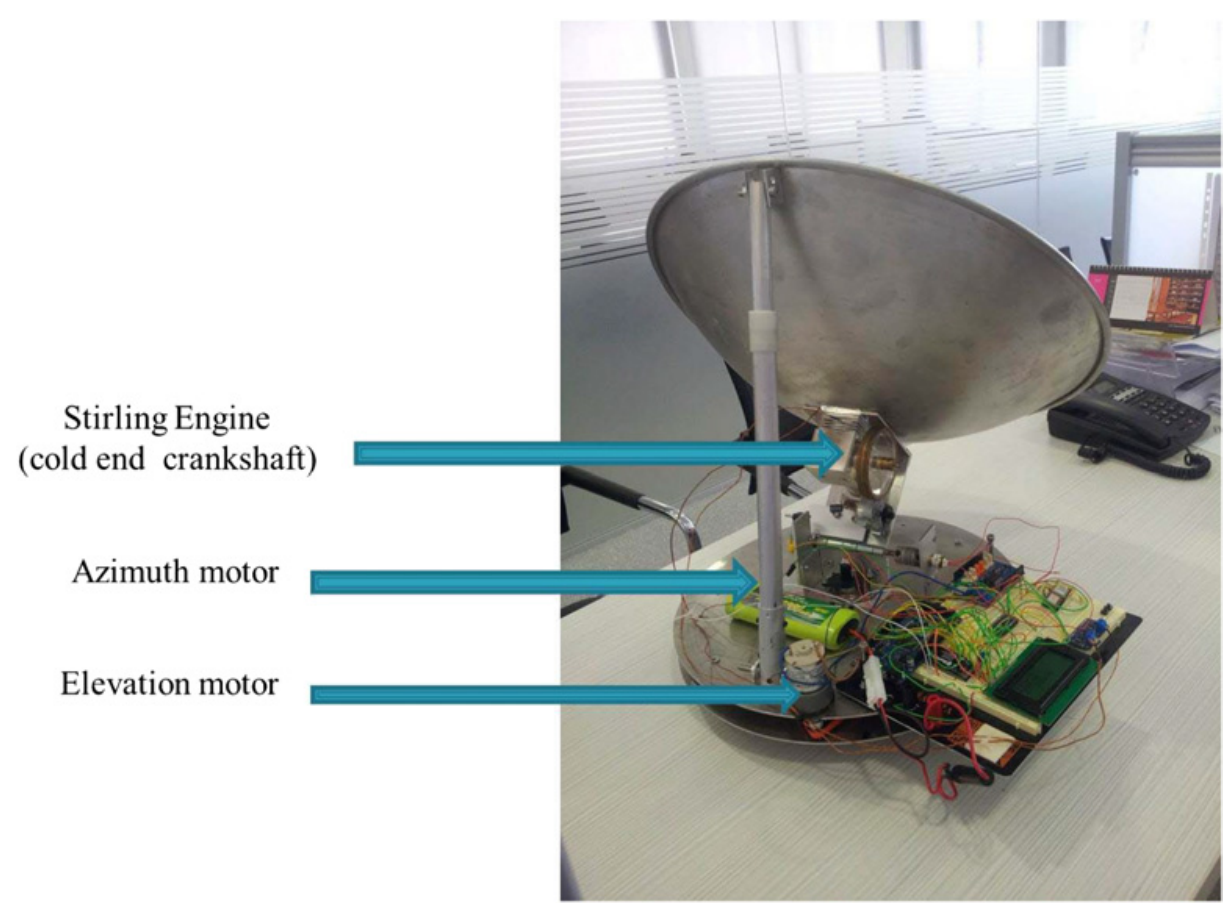

Figure 4. The Sun Tracking System.

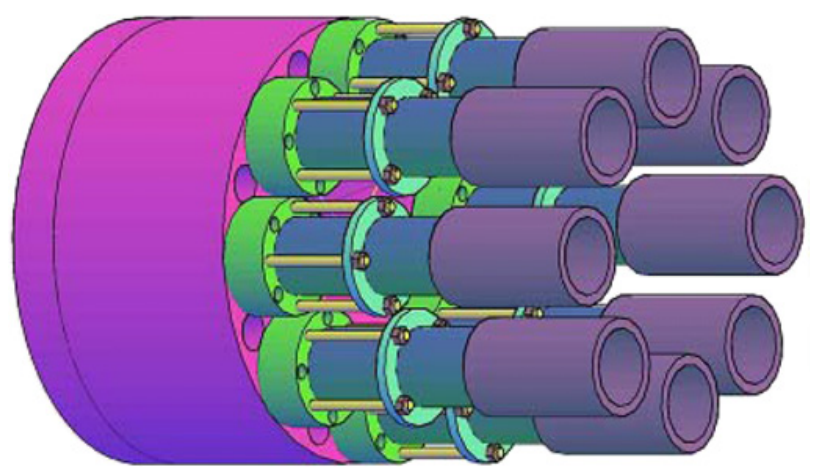

Figure 5. Design of the Heat Collector with the multi-cylinder Stirling Engine.

devices in case of controller failure, in the form of limit switches. To reduce the energy consumed by the motors, the position of the dish is adjusted every 10 minutes.

At the center of the concentrating dish (see Fig. 2) we have the heat collector where the Innovative Stirling Engine resides. In our design of the Stirling Engine (see close up in Fig. 5), conversion of linear motion to circular is carried out with patent developed by the Authors, which results in significant increase of efficiency. The innovative design of this new type of Stirling engine is multi-cylinder without a crank shaft thus greatly reducing friction as well as decreasing the wear out of mechanical parts. 


\section{Efficient conversion of stirling engine output to usable work}

Significant gains in efficiency are also achieved by the utilization of our mechanism for the transmission of linear reciprocating motion of variable amplitude into circular unidirectional motion on a perpendicular axis. Even though there are a number of mechanisms for the transmission of linear, or semi-linear reciprocating motion, to circular motion utilising gears or belts, there are several problems related to such existing mechanisms. The most important problem is that the work being done follows a sinusoidal pattern due to the fact that in most applications the driving force cannot always and continuously be tangential to the circle of rotation. In order to maximise the rate of producing work many applications limit the displacement angle in a reciprocating motion. What is more, in most existing mechanisms, work is produced during half the cycle whereas the other half (return) is usually not producing work. Further, induced vibration is also a common problem in existing systems. Through our approach we can provide a means of utilising reciprocating linear motion of random amplitude to produce continuous circular motion where work can be produced in both forward and reverse direction of the driving force gaining maximum torque from the application of the driving force and therefore gaining the maximum possible rate of work production which can then be transformed into electricity. Further, our approach provides a means of utilising reciprocating linear motion of random amplitude to produce continuous circular motion such that frictional and other energy losses are minimal, and there is no induced vibration.

Also our approach provides a means of utilising reciprocating linear motion of random amplitude to produce continuous circular motion which can consist of simple, readily available parts, and can be manufactured at very low cost. Finally, our model utilise reciprocating linear motion of random amplitude to produce continuous circular motion such it is possible the desired revolutions of the load can be stepped up or stepped down in a simple manner without the use of several gears.

The mechanism for the transmission of lineal reciprocating motion of variable amplitude into circular motion utilises a flexible member such as a wire to transmit the motion, and comprises of three threaded drums, two of which are mounted along the same axis, which transfers motion to the load. Of the two drums, which are mounted along the same axis, which transfers motion to the load, one has a left-hand thread and the other has a right-hand thread and these are mounted on the load axis through roller clutch bearings so that motion is continuously transferred to the load both during forward movement of the driving force and during reverse movement (return) of the driving force. A third threaded drum serves as a guide drum to change the direction of the cable. Its length.is longer than the length of the length of the two other drums together, and its axis is parallel to the axis upon which the two other drums are mounted. This is the axis along which the load is being driven.

Carrying out some preliminary tests using the mechanism described above, we managed to increase the efficiency as shown by the torque gains in Fig. 6. The significant gains in efficiency are shown by the tangential Torque of our design as compared to the crankshaft torque at all displacement levels.

\section{Conclusions and future work}

Economic and social development must be accomplished sustainably, and the solar energy alternative provides a great opportunity in this direction. Through our proposed approach, which is manifested in the HelioTrope prototype we were able to integrate solar energy components into a unique system that efficiently captures and converts solar energy into usable electricity. Specifically through the HelioTrope Model we have managed to implement:

- A holistic approach to develop an innovative solution to solar power production.

- An innovative Prototype that captures the rays of sun at with maximum efficiency

- An Innovative Stirling Engine Design especially developed for Solar Energy transfer to electricity, which greatly improve the system efficiency and effectiveness. 


\section{Torque Vs Displacement}

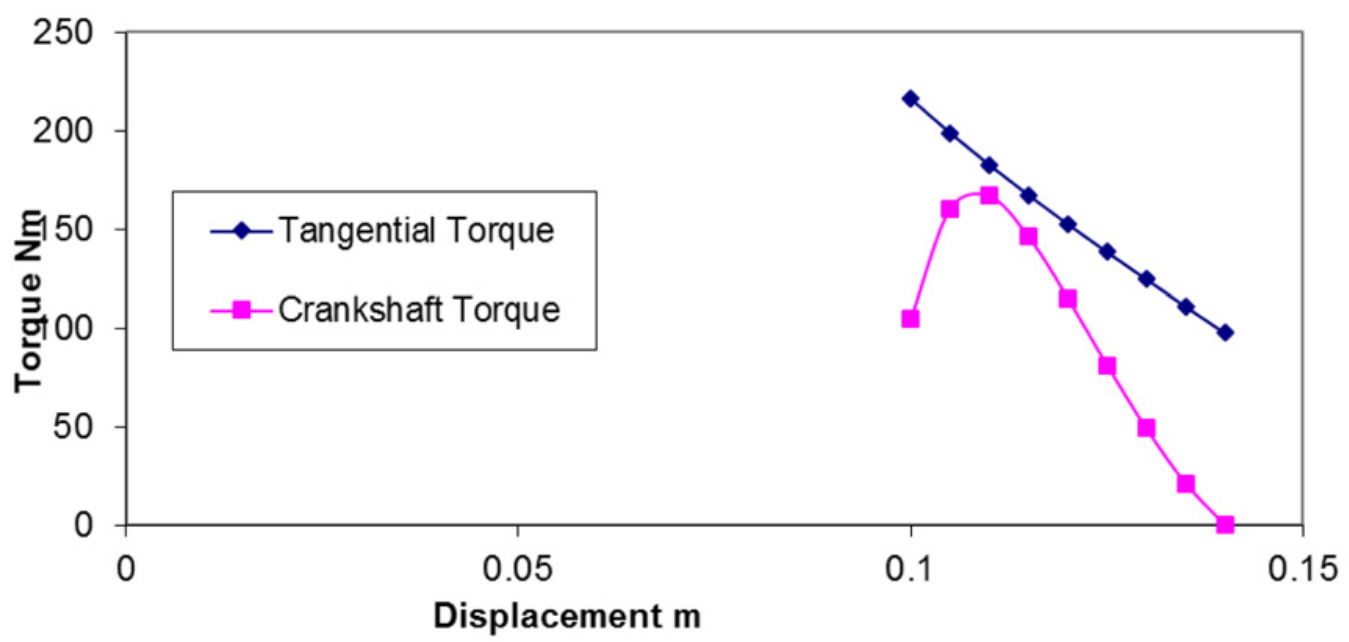

Figure 6. The HelioTrope prototype showing significant gains in Torque.

- A design for the implementation of a patent developed by the authors to convert linear motion into circular for electricity production, which gives a significant increase in efficiency and reduce maintenance costs.

The above results directly contribute to the innovative use of low-carbon energy technologies and to the development of the PV and CSP sector in Research and Development. What remains to be done is to further test the developed HelioTrope prototype in the field to verify the promising preliminary results of significant increase in efficiency.

\section{References}

[1] Showstack, R., Carbon dioxide tops 400 ppm at Mauna Loa, Hawaii, Eos Trans. AGU, 94(21), 192 (2013)

[2] William H. Bloomberg unveils initiative to protect New York City from climate change, The Lookout, http://news.yahoo.com/blogs/lookout/bloomberg-unveils-initiative-protectyork-city-climate-change-205758671.html (2013)

[3] Crabtree, George W., and Nathan S. Lewis. Solar energy conversion. Physics today 60.3 (2007): $37-42$

[4] Hadjixenofontos, S. A mechanism for the transmission of reciprocating motion of variable amplitude into unidirectional circular motion, World Patent WO2005010403A1 (2005)

[5] Papageorgiou, G., Maimaris, A., Hadjixenofontos, S., A Holistic Approach to Solar Energy Production and Management for Sustainable Development, Energy for Sustainability Conference 2013, Coimbra, Portugal, September 9, (2013)

[6] Reda, I.; Andreas, A. Solar Position Algorithm for Solar Radiation Applications. 55 pp.; NREL Report No. TP-560-34302, Revised January (2008) 